\title{
Prophylactic central lymph node dissection performed selectively with cNO papillary thyroid carcinoma according to a risk-scoring model
}

\author{
Xiang Zhong ${ }^{1 \# \wedge}$, Yunpeng Lu ${ }^{1 \#}$, Xu Yin ${ }^{2}$, Quhui Wang ${ }^{1}$, Feiran Wang ${ }^{1}, Z_{\text {Zhixian }} \mathrm{He}^{1}$ \\ ${ }^{1}$ Department of General Surgery, Affiliated Hospital of Nantong University, Nantong, China; ${ }^{2}$ Department of Hepatobiliary and Pancreatic Surgery, \\ Changzhou No.2 People's Hospital Affiliated to Nanjing Medical University, Changzhou, China \\ Contributions: (I) Conception and design: X Zhong, Z He; (II) Administrative support: Z He; (III) Provision of study materials or patients: Y Lu; (IV) \\ Collection and assembly of data: X Yin; (V) Data analysis and interpretation: Q Wang, F Wang; (VI) Manuscript writing: All authors; (VII) Final \\ approval of manuscript: All authors. \\ \#These authors contributed equally to this work. \\ Correspondence to: Zhixian He. Department of General Surgery, Affiliated Hospital of Nantong University, 20 Xisi Road, Nantong 226000 , China. \\ Email: hezhixiangs@sina.com.
}

\begin{abstract}
Background: This study aimed to explore the risk factors of central lymph node metastasis (CLNM) in patients with clinical central lymph node-negative papillary thyroid carcinoma (PTC), and emphasize the guidance of the risk scoring model for prophylactic central lymph node dissection (pCLND) in patients with clinical lymph node-negative (cN0) PTC.

Methods: A total of 582 patients with cN0 PTC who underwent unilateral/bilateral thyroidectomy and prophylactic central lymph node dissection (pCLND) in the Affiliated Hospital of Nantong University from January 2020 to February 2021 were retrospectively analyzed. Univariate and multivariate analyses were performed to determine the risk factors of cN0 PTC. According to the independent risk factors of patients with cN0 PTC, a risk-scoring model was established. Then, the rationality of this risk scoring model was verified by additional clinical data of 112 patients with cN0 PTC in the Affiliated Hospital of Nantong University from March 2021 to April 2021.

Results: Among 582 cases of cN0 PTC, 53.6\% of the patients with cN0 had CLNM. The independent risk factors for CLNM in patients with $\mathrm{cN} 0$ PTC included male gender, $<45$ years of age, tumor with a maximum diameter of $\geq 1.0 \mathrm{~cm}$, tumor location: middle/lower poles of the thyroid gland, multifocality, and extrathyroidal extension (ETE), and some ultrasound features, such as intra-nodular vascularity, microcalcification, irregular shape, and infiltrative margin. According to independent risk factors, a 24-point risk scoring model was established to predict CLNM in patients with cN0 PTC.

Conclusions: Currently, prophylactic central neck lymph node dissection is a controversial operation, which should be selectively performed only for high-risk patients with cN0 PTC. For cN0 PTC patients with scores $\geq 14$ and high-risk patients, even if no CLNM is found before surgery, routine prophylactic CLND is recommended. In addition, for cN0 PTC patients with a score of fewer than 14 points, it is recommended to perform fine-needle aspiration (FNA) before surgery, carefully assess the condition of the central lymph nodes, and then select the best surgical plan based on the results of the assessment.
\end{abstract}

Keywords: Papillary thyroid carcinoma (PTC); risk scoring model; prophylactic central lymph node dissection (pCLND)

^ ORCID: 0000-0002-6636-8962. 
Submitted Dec 07, 2021. Accepted for publication Feb 11, 2022.

doi: 10.21037 /gs-21-906

View this article at: https://dx.doi.org/10.21037/gs-21-906

\section{Introduction}

Thyroid cancer is the most common cancer of the endocrine system, which can be divided into papillary carcinoma, follicular carcinoma, medullary carcinoma, and undifferentiated carcinoma $(1,2)$. A variety of risk factors are considered to be associated with the occurrence of thyroid cancer, such as genetics, radiation, excessive iodine intake, smoking and obesity. Papillary thyroid carcinoma (PTC) and follicular thyroid carcinoma are also called differentiated thyroid carcinoma. The PTC subtype accounts for about $80.0 \%(2,3)$ of thyroid malignant tumors. The incidence of central lymph node metastasis (CLNM) in patients with PTC can reach $40-90 \%$. The main risk factor of a high recurrence rate in patients with PTC is CLNM (4). In general, lymph node metastasis of PTC firstly occurs in the central cervical lymph nodes, and then expands to the lateral cervical lymph nodes, but also has the condition of skip metastasis (5). With the increasing incidence rate in China, the understanding of PTC is deepening in the medical field. Prophylactic central lymph neck dissection (pCLND) implies the removal of the lymph nodes of the central neck compartment in the absence of preoperative clinical or radiologic or intraoperative evidence of pathologic lymphadenopathy (6). At present, if patients with differentiated thyroid cancer have CLNM, it is recommended to perform central lymph node dissection. However, whether to routinely perform prophylactic central neck dissection in patients with clinical central lymph node-negative (cN0) PTC is still an important point of contention (7). The efficacy of prophylactic central neck dissection for $\mathrm{cN} 0 \mathrm{PTC}$ is still uncertain (7-9), and the incidence of complications after prophylactic central cervical lymph node dissection is high, such as recurrent laryngeal nerve injury, temporary/ permanent hypoparathyroidism $(10,11)$, and chyle leakage (12). Therefore, it is necessary to clarify the predictors of CLNM in patients with cN0 PTC and to selectively perform prophylactic lymph node dissection in high-risk patients. In this article, we retrospectively analyzed the clinicopathological data of 582 patients with cN0 PTC in the Affiliated Hospital of Nantong University, and calculated the risk factors of CLNM. Finally, we established a 24-point risk scoring model based on the risk factors of CLNM.
According to this risk scoring model, we can predict the highrisk population of patients with $\mathrm{cN} 0 \mathrm{PTC}$ who may develop CLNM, which assists the formulation of a reasonable surgical plan for them to achieve the best treatment effect. We present the following article in accordance with the STARD reporting checklist (available at https://gs.amegroups.com/ article/view/10.21037/gs-21-906/rc).

\section{Methods}

\section{Patients}

The study was conducted in accordance with the Declaration of Helsinki (as revised in 2013). This study was approved by the Ethics Committee of Affiliated Hospital of Nantong University (ID: 2013-67). Informed consent was provided by all participants. We included patients who underwent their first thyroid surgery at the General Surgery Department of the Affiliated Hospital of Nantong University from January 2020 to April 2021 and were confirmed to have cN0 PTC. We excluded patients with the following criteria from the study: other types of thyroid malignancies, clinical lymph node-positive PTC, had not undergone neck central lymph node dissection, a history of thyroid surgery, and those with incomplete data. A total of 694 eligible patients were collected for the study, among whom 582 were included in the model establishment study and 112 were included in the model test study.

\section{Surgical procedures}

All patients underwent ultrasound and cervical contrastenhanced computed tomography (CT) examination before surgery to identify thyroid nodules and cervical lymph nodes. In this study, FNA was not a routine operation and was only performed on a small number of patients. All pathological sections of tumors were histologically examined during the operation. Patients with bilateral PTC underwent total thyroidectomy plus bilateral central lymph node dissection. Patients with unilateral PTC underwent total thyroidectomy plus bilateral central lymph node dissection (CLND) or unilateral lobectomy plus isthmectomy and ipsilateral CLND based on the status of 
the primary tumor. When patients with unilateral PTC met 1 or more of the following conditions, we considered performing lobectomy plus isthmectomy: tumor $>4 \mathrm{~cm}$; positive resection margins; gross extrathyroidal extension; macroscopic multifocal disease $(>1 \mathrm{~cm})$; macroscopic nodal metastasis; vascular invasion.

\section{Clinicopathological properties}

By collecting the following clinicopathological characteristics of patients with cN0 PTC, we explored the risk factors for CLNM: gender, age, tumor volume and number, tumor location in the thyroid gland, extrathyroidal extension, Hashimoto's thyroiditis (HT), and some ultrasound features, including intra-nodular vascularity, microcalcification, aspect ratio $>1$, irregular shape, and infiltrative margin. For postoperative pathological specimens, 2 or more experienced pathologists conducted re-examination and cross-examination. We defined the presence of 2 or more papillary carcinoma lesions in the thyroid as multifocality. Papillary thyroid microcarcinoma was defined as PTC with a maximum diameter of $<1 \mathrm{~cm}$. Extrathyroid extension (ETE) referred to a primary tumor of PTC that extended to the surrounding tissues of the thyroid through the thyroid capsule, invaded anterior cervical muscles, sternocleidomastoid muscle, larynx, trachea, esophagus, recurrent laryngeal nerve, and even the internal jugular vein and common carotid artery. According to the location of the tumor, we divided PTC into three types. The location of the tumor above the isthmus plane is divided as upper polar group. The location of the tumor parallel to the isthmus plane is defined as middle polar group. The location of the tumor below the isthmus plane is divided as lower polar group. If the main lesion occupies two adjacent parts, it is divided according to two-thirds of volume the lesion volume. Patients diagnosed with HT must meet any of the following three criteria, such as anti-thyroid peroxidase (TPO) antibody-positive; antithyroglobulin positive; or pathological confirmation of HT.

\section{Statistical analysis}

All statistical analyses were conducted using 25 version SPSS software (IBM Corp., Chicago, IL, USA) and R language (https://www.r-project.org/). Among them, continuous variables were expressed as mean \pm standard deviation. For the variables of patients with $\mathrm{cN} 0$ PTC with or without CLNM, Fisher's exact test and chi-square test were used for univariate analysis. Then, the variables with $\mathrm{P}<0.05$ in the results of the univariate analysis were included in the multivariate logistic regression analysis to calculate the risk factors for the central lymph nodes metastasis, and a forest plot of the results of the multivariate analysis was drawn with $\mathrm{R}$ language. According to the results of multivariate analysis, a risk-scoring model was constructed to calculate the incidence of CLNM. We selected independent risk factors for CLNM as scoring indicators. According to the beta coefficient obtained by the logistic regression model, the scores of each risk factor were weighted. To simplify the scoring model, all coefficients were divided by the smallest one and then rounded to the nearest integer. The total score of each risk factor of the patient represented his/her total score. A receiver operating characteristic (ROC) curve was used to evaluate the predictive effect of the risk scoring model, and a suitable cut-off point was found.

\section{Results}

\section{Basic clinicopathological characteristics of the patients used to establish the model}

This study summarized the baseline clinicopathological characteristics of patients with cN0 PTC to establish a CLNM risk scoring model (Table 1). Among the 582 patients with PTC, 138 cases were males and 444 cases were females, with an average age of $46 \pm 12$ years old (18-77 years old). The maximum tumor diameter was $0.10-5.50 \mathrm{~cm}$, and the average maximum diameter was $1.16 \pm 0.84 \mathrm{~cm}$. A total of 125 cases $(21.5 \%)$ had tumors located in the upper pole of the thyroid, and 457 cases $(78.5 \%)$ were located in the middle/lower poles of the thyroid. There were 67 patients (11.5\%) with extrathyroidal extension. Among the patients, 115 had HT, accounting for $19.8 \%$. There were 422 cases with isolated PTC and 160 patients with multiple PTC. Thyroid ultrasound showed 382 patients with intra-nodular vascularity, 479 patients with microcalcification, 184 patients with aspect ratio $>1,433$ patients with an irregular shape, and 458 patients with infiltrative margins. All cases with cN0 PTC underwent prophylactic central lymph node dissection, and 312 cases $(53.6 \%)$ of central lymph node metastases were found. Surgical complications included the following: 31 cases of temporary hypoparathyroidism $(5.3 \%), 5$ cases of permanent hypoparathyroidism $(0.9 \%)$, 9 cases of unilateral vocal cord paralysis (1.5\%), 2 cases of bilateral vocal cord paralysis $(0.3 \%), 3$ cases of postoperative bleeding $(0.5 \%)$, and 4 cases of chyle leakage $(0.7 \%)$. 
Table 1 Demographics and clinical characteristics of patients undergoing central lymph node dissection in creating a model group

\begin{tabular}{|c|c|c|c|}
\hline \multirow{2}{*}{$\begin{array}{l}\text { Clinicopathological } \\
\text { properties }\end{array}$} & \multirow{2}{*}{ Total } & \multicolumn{2}{|c|}{ Central lymph node metastasis } \\
\hline & & No, n (\%) & Yes, n (\%) \\
\hline Subject & 582 & $270(46.4)$ & $312(53.6)$ \\
\hline \multicolumn{4}{|l|}{ Gender } \\
\hline Female & 444 & $238(53.6)$ & $206(46.4)$ \\
\hline Male & 138 & $32(23.2)$ & $106(76.8)$ \\
\hline \multicolumn{4}{|l|}{ Age (years) } \\
\hline$<45$ & 243 & $91(37.4)$ & $152(62.6)$ \\
\hline$\geq 45$ & 339 & $179(52.8)$ & $160(47.2)$ \\
\hline \multicolumn{4}{|l|}{ Tumor size $(\mathrm{cm})$} \\
\hline$<1$ & 306 & $205(67.0)$ & $101(33.0)$ \\
\hline$\geq 1$ & 276 & $65(23.6)$ & $211(76.4)$ \\
\hline \multicolumn{4}{|l|}{ Tumor location } \\
\hline Upper & 125 & $100(80.0)$ & $25(20.0)$ \\
\hline Middle/lower & 457 & $170(37.2)$ & $287(62.8)$ \\
\hline \multicolumn{4}{|l|}{ Ultrasound feature } \\
\hline \multicolumn{4}{|c|}{ Intra-nodular vascularity } \\
\hline No & 200 & $137(68.5)$ & $63(31.5)$ \\
\hline Yes & 382 & $133(34.8)$ & $249(65.2)$ \\
\hline \multicolumn{4}{|l|}{ Microcalcification } \\
\hline No & 103 & $79(76.7)$ & $24(23.3)$ \\
\hline Yes & 479 & $191(39.9)$ & $288(60.1)$ \\
\hline \multicolumn{4}{|l|}{ Aspect ratio $>1$} \\
\hline No & 398 & $185(46.5)$ & $213(53.5)$ \\
\hline Yes & 184 & 85 (46.2) & $99(53.8)$ \\
\hline \multicolumn{4}{|l|}{ Irregular shape } \\
\hline No & 149 & $116(77.9)$ & $33(22.1)$ \\
\hline Yes & 433 & $154(35.6)$ & $279(64.4)$ \\
\hline \multicolumn{4}{|l|}{ Infiltrative margin } \\
\hline No & 124 & $101(81.5)$ & $23(18.5)$ \\
\hline Yes & 458 & $169(36.9)$ & $289(63.1)$ \\
\hline \multicolumn{4}{|l|}{ Multifocality } \\
\hline No & 422 & $212(50.2)$ & $210(49.8)$ \\
\hline Yes & 160 & $58(36.3)$ & $102(63.7)$ \\
\hline
\end{tabular}

Table 1 (continued)
Table 1 (continued)

\begin{tabular}{lccc}
\hline \multirow{2}{*}{$\begin{array}{l}\text { Clinicopathological } \\
\text { properties }\end{array}$} & Total & \multicolumn{2}{c}{ Central lymph node metastasis } \\
\cline { 3 - 4 } & & No, n (\%) & Yes, n (\%) \\
\hline Extrathyroidal extension & & & \\
No & 515 & $265(51.5)$ & $250(48.5)$ \\
Yes & 67 & $5(7.5)$ & $62(92.5)$ \\
Hashimoto's thyroiditis & & & \\
No & 467 & $223(47.8)$ & $244(52.2)$ \\
Yes & 115 & $47(40.9)$ & $68(59.1)$ \\
\hline
\end{tabular}

\section{Correlation between clinicopathological factors and lymph node metastasis in the central area of the neck}

In 582 (cN0) PTC patients who received prophylactic central lymph node dissection (pCLND), we analyzed the risk factors for CLNM. Univariate analysis showed that gender, age, tumor volume and number, tumor location in the glands, extrathyroidal extension, and ultrasound features, including intra-nodular vascularity, microcalcification, irregular shape, and infiltrative margin were significantly correlated with CLNM $(\mathrm{P}<0.05)$. However, there was no significant correlation with aspect ratio $>1$ or HT (Table 2). Multivariate analysis showed that male gender [odds ratio (OR): $5.315,95 \%$ confidence interval (CI): 2.881 to $9.806, \mathrm{P}<0.001$ ), age $<45$ years old (OR: $1.792,95 \%$ CI: 1.104 to $2.907, \mathrm{P}=0.018$ ); maximum diameter of the tumor $\geq 1.0 \mathrm{~cm}$ (OR: $7.757,95 \%$ CI: 4.716 to $12.760, \mathrm{P}<0.001)$; tumor located in the middle/lower pole of the thyroid gland (OR: 8.102, 95\% CI: 4.213 to 15.582 , $\mathrm{P}<0.001$ ); multifocality (OR: $1.844,95 \%$ CI: 1.079 to 3.153 , $\mathrm{P}=0.025$ ); extrathyroidal extension (OR: 5.875 , 95\% CI: 1.953 to $17.674, \mathrm{P}=0.002)$; and ultrasound showing intranodular vascularity (OR: $2.570,95 \%$ CI: 1.569 to 4.209 , $\mathrm{P}=0.001$ ), microcalcification (OR: $2.639,95 \% \mathrm{CI}: 1.354$ to $5.141, \mathrm{P}=0.004)$, irregular shape (OR: 2.398 , $95 \% \mathrm{CI}$ : 1.199 to $4.794, \mathrm{P}=0.013)$, and infiltrative margin (OR: 2.559, 95\% CI: 1.202 to $5.447, \mathrm{P}=0.015)$ were all independent risk factors for CLNM $(\mathrm{P}<0.05)$ (Table 3, Figure 1).

\section{Establishment of a risk scoring model for predicting CLNM}

In our study, according to the beta coefficients of 10 independent risk factors: (male, age $<45$ years old, the maximum diameter of tumor $\geq 1.0 \mathrm{~cm}$, tumor located in 
Table 2 Univariate analysis of risk factors for central lymph node metastasis

\begin{tabular}{|c|c|c|c|}
\hline Risk factors & OR & $95 \% \mathrm{Cl}$ & $P$ value \\
\hline Gender (male vs. female) & 3.827 & $2.472-5.925$ & $<0.001$ \\
\hline \multicolumn{4}{|l|}{ Age (years) } \\
\hline$<45$ & 1.869 & $1.335-2.615$ & $<0.001$ \\
\hline$\geq 45$ & 1 & & \\
\hline \multicolumn{4}{|l|}{ Tumor size $(\mathrm{cm})$} \\
\hline$<1$ & 1 & & \\
\hline$\geq 1$ & 6.589 & $4.569-9.502$ & $<0.001$ \\
\hline \multicolumn{4}{|l|}{ Tumor location } \\
\hline Upper & 1 & & \\
\hline Middle/lower & 6.753 & $4.189-10.887$ & $<0.001$ \\
\hline \multicolumn{4}{|l|}{ Ultrasound feature } \\
\hline $\begin{array}{l}\text { Intra-nodular vascularity } \\
\text { (yes vs. no) }\end{array}$ & 4.07 & $2.83-5.87$ & $<0.001$ \\
\hline Microcalcification (yes vs. no) & 4.96 & $3.03-8.12$ & $<0.001$ \\
\hline Aspect ratio >1 (yes vs. no) & 1.012 & $0.713-1.436$ & 1.000 \\
\hline Irregular shape (yes vs. no) & 6.37 & $4.13-9.83$ & $<0.001$ \\
\hline Infiltrative margin (yes vs. no) & 7.55 & $4.62-12.35$ & $<0.001$ \\
\hline Multifocality (yes vs. no) & 1.775 & $1.221-2.58$ & 0.003 \\
\hline $\begin{array}{l}\text { Extrathyroidal extension } \\
\text { (yes vs. no) }\end{array}$ & 13.14 & $5.20-33.23$ & $<0.001$ \\
\hline $\begin{array}{l}\text { Hashimoto's thyroiditis } \\
\text { (yes vs. no) }\end{array}$ & 1.322 & $0.874-2.000$ & 0.211 \\
\hline
\end{tabular}

OR, odds ratio; $\mathrm{Cl}$, confidence interval.

the middle/lower pole of the thyroid gland, multifocality, extrathyroidal extension, and ultrasound features, including intra-nodular vascularity, microcalcification, irregular shape, and infiltrative margin) in the multivariate analysis of CLNM in patients with $\mathrm{cNO}$ PTC, a 24 point risk scoring model was constructed (Table 4). According to the scoring model, the positive rate of CLNM in cN0 PTC patients ranged from 0 to $100 \%$ (Table 5). The ROC curve of the CLNM risk scoring model was drawn. The area under the curve (AUC) of the model for predicting CLNM was 0.904 (95\% CI: 0.880 to $0.928, \mathrm{P}<0.001)$ (Figure 2), indicating that the discriminant ability of the model was acceptable. In addition, the total score $=14$ was used as an appropriate cutoff point for the model; patients with a total score between 0 and 13 were divided into the CLNM low-risk group
Table 3 Multivariate analysis of risk factors for central lymph node metastasis

\begin{tabular}{lccc}
\hline Risk factors & OR & $95 \% \mathrm{Cl}$ & $\mathrm{P}$ value \\
\hline Gender (male vs. female) & 5.315 & $2.881-9.806$ & $<0.001$ \\
Age (years) & & & \\
$<45$ & 1.792 & $1.104-2.907$ & 0.018 \\
$\geq 45$ & & & \\
Tumor size (cm) & & & \\
$<1$ & & & \\
$\geq 1$ & 7.757 & $4.716-12.760$ & $<0.001$ \\
Tumor location & & & \\
Upper & & & \\
Middle/lower & 8.102 & $4.213-15.582$ & $<0.001$ \\
Ultrasound feature & & & \\
Intra-nodular vascularity & 2.570 & $1.569-4.209$ & $<0.001$ \\
(yes vs. no) & & & 0.0025 \\
Microcalcification (yes vs. no) & 2.639 & $1.354-5.141$ & 0.004 \\
Irregular shape (yes vs. no) & 2.398 & $1.199-4.794$ & 0.013 \\
Infiltrative margin (yes vs. no) & 2.559 & $1.202-5.447$ & 0.015 \\
Multifocality (yes vs. no) & 1.844 & $1.079-3.153$ & 0.025 \\
Extrathyroidal extension & 5.875 & $1.953-17.674$ & 0.002 \\
(yes vs. no) & & & \\
\hline OR, odds ratio; Cl, confidence interval. & & \\
\hline
\end{tabular}

(average CLNM rate: $23.04 \%$ ), and patients with a score between 14 and 24 were divided into the high-risk group of CLNM (average CLNM rate: 87.96\%).

\section{The basic clinicopathological characteristics of patients used to test the risk scoring model and verify the rationality of the model}

According to the same admission criteria (Table 6), from March to April 2021, 112 patients with cN0 PTC were screened into the verification group from the Nantong University Affiliated Hospital. Among 112 patients, 21 cases were males and 91 cases were females, with an average age of $47 \pm 12$ years (23-79 years). The maximum diameter of the tumor was $0.10-7.00 \mathrm{~cm}$, and the average maximum diameter was $1.23 \pm 1.04 \mathrm{~cm}$. A total of 42 cases $(37.5 \%)$ had tumors located in the upper pole of the thyroid, and 70 cases $(62.5 \%)$ were located in the middle/lower poles of 


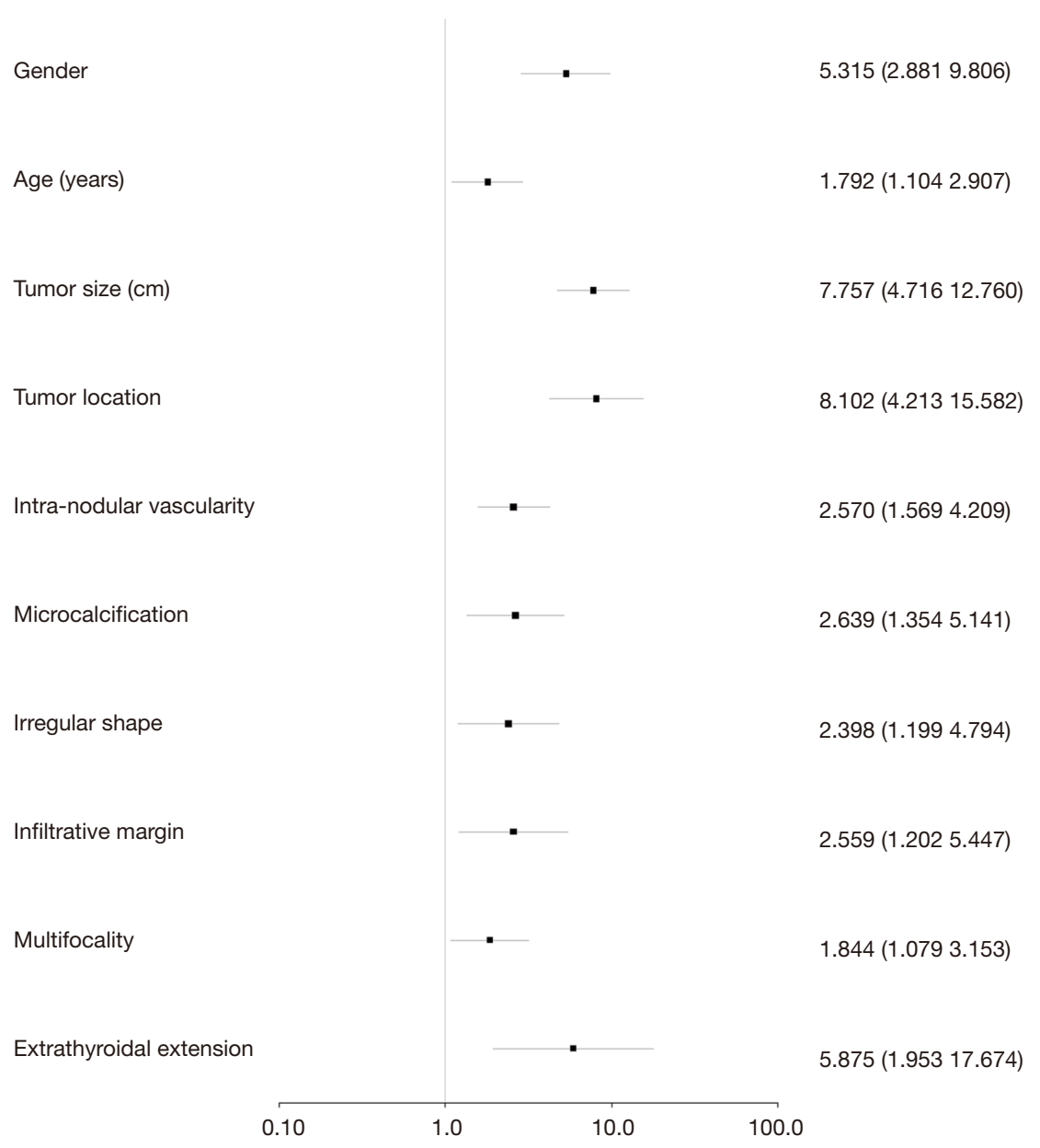

Figure 1 Forest map of multivariate analysis.

the thyroid. There were 10 cases $(8.9 \%)$ with extrathyroidal extension. There were 80 patients with isolated PTC and 32 patients with multiple PTC. Thyroid ultrasound showed 67 patients with intra-nodular vascularity, 71 patients with microcalcification, 62 patients with irregular shape, and 74 patients with infiltrative margin. All patients with cN0 PTC underwent prophylactic central lymph node dissection, and 58 cases $(51.8 \%)$ of CLNM were found. According to the scoring table, the clinical case characteristics of the patients in the test group were converted into parameters and substituted into the risk scoring model to calculate the probability of CLNM in each patient as the risk score. Postoperative pathology was used as the gold standard. In the end, the area under the ROC curve obtained in this study was 0.956 (95\% CI: 0.915 to $0.996, \mathrm{P}<0.001)$ (Figure 3). Therefore, the risk scoring model for CLNM has an excellent predictive effect.

\section{Discussion}

Due to the increasing incidence of PTC, thyroid diseases have gradually attracted increasing attention. According to US medical and health statistics, in recent years, the incidence of thyroid cancer has steadily risen (about 4\% per year). It has become the fastest-increasing malignancy of all major cancers in the US and has tripled in the past 3 decades (13). With the advancement of ultrasound technology, ultrasound examination can provide a clearer description and judgment of the nature of thyroid nodules, and can better observe the condition of the central lymph nodes (14). Regarding the treatment of PTC, especially cN0 PTC, whether prophylactic central cervical lymph node dissection is necessary has remained a controversial topic (7). According to the latest guidelines of the National Comprehensive Cancer Network (NCCN), patients with PTC who are clinically positive or have lymph 
Table 4 Development of a 24-point risk-scoring model to predict central lymph node metastasis

\begin{tabular}{|c|c|c|c|}
\hline Variables & $P$ value & Beta coefficient & Point \\
\hline \multicolumn{4}{|l|}{ Gender } \\
\hline \multicolumn{4}{|l|}{ Female } \\
\hline Male & $<0.001$ & 1.671 & 3.000 \\
\hline \multicolumn{4}{|l|}{ Age (years) } \\
\hline$<45$ & 0.018 & 0.583 & 1.000 \\
\hline \multicolumn{4}{|l|}{$\geq 45$} \\
\hline \multicolumn{4}{|l|}{ Tumor size (cm) } \\
\hline \multicolumn{4}{|l|}{$<1$} \\
\hline$\geq 1$ & $<0.001$ & 2.049 & 4.000 \\
\hline \multicolumn{4}{|l|}{ Tumor location } \\
\hline \multicolumn{4}{|l|}{ Upper } \\
\hline Middle/lower & $<0.001$ & 2.092 & 4.000 \\
\hline \multicolumn{4}{|c|}{ Ultrasound feature } \\
\hline \multicolumn{4}{|c|}{ Intra-nodular vascularity } \\
\hline \multicolumn{4}{|l|}{ Absence } \\
\hline Presence & $<0.01$ & 0.944 & 2.000 \\
\hline \multicolumn{4}{|c|}{ Microcalcification } \\
\hline \multicolumn{4}{|l|}{ Absence } \\
\hline Presence & 0.004 & 0.970 & 2.000 \\
\hline \multicolumn{4}{|c|}{ Irregular shape } \\
\hline \multicolumn{4}{|l|}{ Absence } \\
\hline Presence & 0.013 & 0.875 & 2.000 \\
\hline \multicolumn{4}{|c|}{ Infiltrative margin } \\
\hline \multicolumn{4}{|l|}{ Absence } \\
\hline Presence & 0.015 & 0.940 & 2.000 \\
\hline \multicolumn{4}{|l|}{ Multifocality } \\
\hline \multicolumn{4}{|l|}{ Absence } \\
\hline Presence & 0.025 & 0.612 & 1.000 \\
\hline \multicolumn{4}{|c|}{ Extrathyroidal extension } \\
\hline \multicolumn{4}{|l|}{ Absence } \\
\hline Presence & 0.002 & 1.771 & 3.000 \\
\hline
\end{tabular}

node metastasis confirmed by biopsy should undergo routine central lymph node dissection. When lymph node metastasis occurs in the central area of the neck, it can
Table 5 Risk scores and percentage of central lymph node metastasis in PTC patient

\begin{tabular}{|c|c|c|c|c|}
\hline Risk score & CLNM (+) & CLNM (-) & Total & Positive rate \\
\hline 0 & 0 & 4 & 4 & $0.0 \%$ \\
\hline 1 & 0 & 3 & 3 & $0.0 \%$ \\
\hline 2 & 0 & 5 & 5 & $0.0 \%$ \\
\hline 3 & 0 & 5 & 5 & $0.0 \%$ \\
\hline 4 & 1 & 13 & 14 & $7.1 \%$ \\
\hline 5 & 0 & 10 & 10 & $0.0 \%$ \\
\hline 6 & 0 & 18 & 18 & $0.0 \%$ \\
\hline 7 & 4 & 23 & 27 & $14.8 \%$ \\
\hline 8 & 3 & 36 & 39 & $7.7 \%$ \\
\hline 9 & 4 & 29 & 33 & $12.1 \%$ \\
\hline 10 & 7 & 29 & 36 & $19.4 \%$ \\
\hline 11 & 9 & 19 & 28 & $32.1 \%$ \\
\hline 12 & 20 & 21 & 41 & $48.8 \%$ \\
\hline 13 & 23 & 22 & 45 & $51.1 \%$ \\
\hline 14 & 33 & 10 & 43 & $76.7 \%$ \\
\hline 15 & 25 & 12 & 37 & $67.6 \%$ \\
\hline 16 & 49 & 3 & 52 & $94.2 \%$ \\
\hline 17 & 43 & 6 & 49 & $87.8 \%$ \\
\hline 18 & 18 & 0 & 18 & $100.0 \%$ \\
\hline 19 & 17 & 1 & 18 & $94.4 \%$ \\
\hline 20 & 35 & 1 & 36 & $97.2 \%$ \\
\hline 21 & 10 & 0 & 10 & $100 \%$ \\
\hline 22 & 4 & 0 & 4 & $100 \%$ \\
\hline 23 & 5 & 0 & 5 & $100 \%$ \\
\hline 24 & 2 & 0 & 2 & $100 \%$ \\
\hline
\end{tabular}

be achieved by unilateral or bilateral dissection of the VI area (15). Based on the results of the previous randomized controlled trial, this trial randomly divided 181 patients with PTC who received total thyroidectomy or total thyroidectomy and central lymph node dissection . There was no difference between the 2 groups (16). Therefore, if PTC proves to be clinically negative in lymph nodes, many expert groups do not recommend prophylactic central lymph node dissection. However, many experts currently 


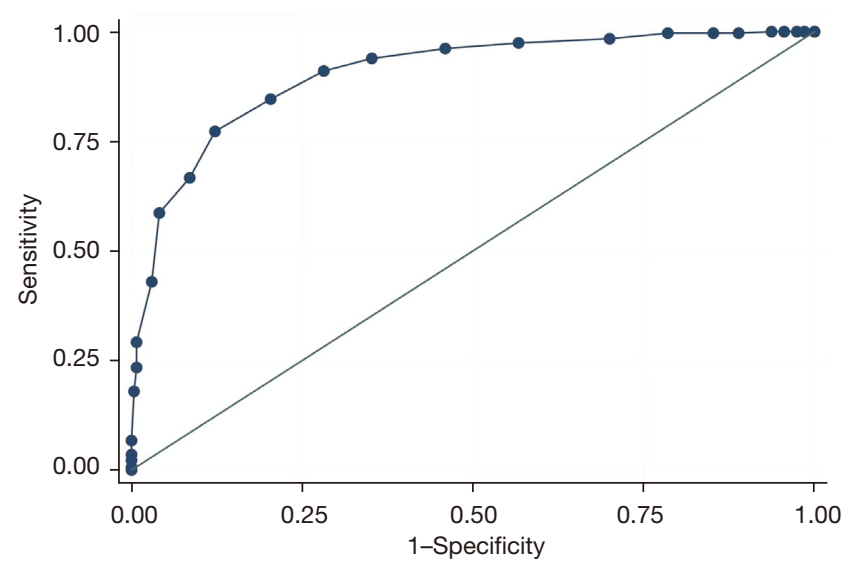

Area under ROC curve $=0.9041$

Figure 2 ROC curve of the ability of risk scoring model to predict the risk of CLNM in creating a model group. ROC, receiver operating characteristic; CLNM, central lymph node metastasis.

support prophylactic central lymph node dissection in patients with cN0 PTC (17), for the following reasons: subclinical CLNM is a common surgical method at present; the evaluation of lymph node metastasis in the central region before operation is subjective; it can reduce the recurrence rate and improve the prognosis to a certain extent; the intervention is helpful for surgeons to carry out clinicopathological staging of thyroid cancer, so as to guide follow-up treatment; for experienced surgeons, pCND can be performed safely in patients with cNO PTC. In this study, the rate of CLNM was detected by routine preventive central lymph node dissection in patients with cN0 PTC. The study found that $53.6 \%$ of patients with cN0 PTC had CLNM. The incidence of lymph node metastasis in the central area of this study is consistent with the 11.7$63.8 \%$ incidence reported in previous studies (18). The complications of central lymph node dissection are serious, including recurrent laryngeal nerve injury, temporary/ permanent hypoparathyroidism, and chyle leakage. In addition, in patients with cN0 PTC, the incidence of CLNM of $53.6 \%$ cannot persuade all cN0 PTC patients to routinely perform prophylactic central cervical lymph node dissection. Therefore, we further studied the clinicopathological and ultrasound characteristics related to CLNM to help screen outpatients with high-risk cN0 PTC who may have CLNM, so as to achieve more precise treatment for them.
Table 6 Demographics and clinical characteristics of patients undergoing central lymph node dissection in the testing model group

\begin{tabular}{|c|c|c|c|}
\hline \multirow{2}{*}{$\begin{array}{l}\text { Clinicopathological } \\
\text { properties }\end{array}$} & \multirow{2}{*}{ Total } & \multicolumn{2}{|c|}{ Central lymph node metastasis } \\
\hline & & No, n (\%) & Yes, n (\%) \\
\hline Patient & 112 & $54(48.2)$ & $58(51.8)$ \\
\hline \multicolumn{4}{|l|}{ Gender } \\
\hline Female & 91 & $51(56.0)$ & $40(44.0)$ \\
\hline Male & 21 & $3(14.3)$ & $18(85.7)$ \\
\hline \multicolumn{4}{|l|}{ Age (years) } \\
\hline$<45$ & 50 & $21(42.0)$ & $29(58.0)$ \\
\hline$\geq 45$ & 62 & $33(53.2)$ & $29(46.8)$ \\
\hline \multicolumn{4}{|l|}{ Tumor size $(\mathrm{cm})$} \\
\hline$<1$ & 58 & $41(70.7)$ & $17(29.3)$ \\
\hline$\geq 1$ & 54 & $13(24.1)$ & $41(75.9)$ \\
\hline \multicolumn{4}{|l|}{ Tumor location } \\
\hline Upper & 42 & $38(90.5)$ & $4(9.5)$ \\
\hline Middle/lower & 70 & $16(22.9)$ & $54(77.1)$ \\
\hline \multicolumn{4}{|l|}{ Ultrasound feature } \\
\hline \multicolumn{4}{|c|}{ Intra-nodular vascularity } \\
\hline No & 45 & $37(82.2)$ & $8(17.8)$ \\
\hline Yes & 67 & $17(25.4)$ & $50(74.6)$ \\
\hline \multicolumn{4}{|l|}{ Microcalcification } \\
\hline No & 41 & $30(73.2)$ & $11(26.8)$ \\
\hline Yes & 71 & $24(33.8)$ & $47(66.2)$ \\
\hline \multicolumn{4}{|l|}{ Irregular shape } \\
\hline No & 50 & $42(84.0)$ & $8(16.0)$ \\
\hline Yes & 62 & $12(19.4)$ & $50(80.4)$ \\
\hline \multicolumn{4}{|l|}{ Infiltrative margin } \\
\hline No & 38 & $33(86.8)$ & $5(13.2)$ \\
\hline Yes & 74 & $21(28.4)$ & $53(71.6)$ \\
\hline \multicolumn{4}{|l|}{ Multifocality } \\
\hline No & 80 & $43(53.8)$ & $37(46.2)$ \\
\hline Yes & 32 & $11(34.4)$ & $21(65.6)$ \\
\hline \multicolumn{4}{|c|}{ Extrathyroidal extension } \\
\hline No & 102 & $54(52.9)$ & $48(47.1)$ \\
\hline Yes & 10 & $0(0.0)$ & $10(100.0)$ \\
\hline
\end{tabular}




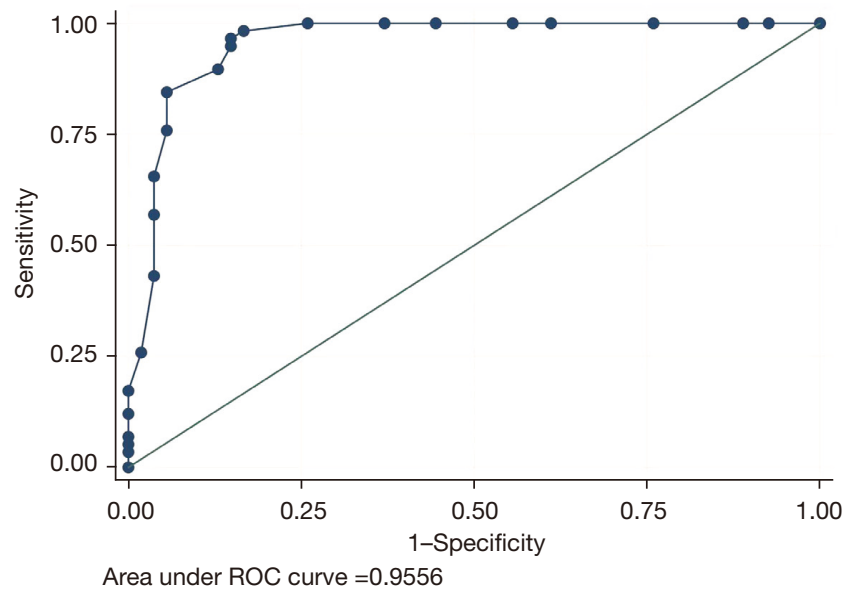

Figure 3 ROC curves of the ability of risk scoring model to predict the risk of CLNM in the testing model group. ROC, receiver operating characteristic; CLNM, central lymph node metastasis; PTC, papillary thyroid carcinoma.

In this study, male gender, $<45$ years of age, tumor with a maximum diameter of $\geq 1.0 \mathrm{~cm}$, tumor location: middle/lower poles of the thyroid gland, multifocality, and extrathyroidal extension were all independent predictors of the CLNM in patients with cN0 PTC. Our results are consistent with previous studies on risk factors for CLNM in patients with PTC (19-23). Different from other risk-scoring models, we have added some objective indicators in preoperative thyroid ultrasound examination to enrich the model, aiming to improve the effectiveness of this model in predicting CLNM in patients with $\mathrm{cN} 0$ PTC. The indiactors include intra-nodular vascularity, microcalcification, irregular shape, infiltrative margin, and aspect ratios $>1$. Surprisingly, the study revealed that intranodular vascularity, microcalcification, irregular shape, and infiltrative margin were all independent predictors of CLNM in patients with cN0 PTC. According to current guidelines, ultrasonography (US) is the main imaging method for preoperative evaluation of cervical lymph node metastasis of thyroid cancer (24). Jang et al. found that the more abundant the blood supply of the tumor, the higher the probability of CLNM (25). In our study, there was a significant difference in blood flow signals between CLNM-positive patients and CLNM-negative patients. The abundance of blood flow signals often means that the blood supply of the tumor is abundant, that is, there is more possibility of lymph node metastasis. Previous studies have shown that large calcifications increase the risk of PTC, and marginal calcifications increase the risk of follicular thyroid carcinoma (FTC) (26). In addition to helping to determine the nature of thyroid nodules, microcalcification can also be used as a risk factor for predicting CLNM in central lymph nodes. In addition, the irregular shape and infiltrative margin of the thyroid nodules also help to make a judgment about the CLNM before surgery, which is consistent with the results of previous studies (27-29). The irregular shape and infiltrative margin can not only be used to judge the nature of thyroid nodules but also help predict CLNM in patients with (cN0) PTC. In the past, people were more inclined to analyze clinicalpathological characteristics instead of relying on ultrasound factors. Part of the reason was the insufficient accuracy of ultrasound technology and the strong subjective factors of ultrasound doctors at that time. However, with the continuous advancement of ultrasound technology, the nodules seen and described on ultrasound examination are more objective. The preoperative description of ultrasound examination has been of great value in the assessment of cervical CLNM in patients with PTC (30). Therefore, the preoperative systematic evaluation of the clinicopathological characteristics and thyroid ultrasound features of patients with cN0 PTC has important predictive significance for determining whether CLNM occurs.

In addition to the study of independent risk factors for CLNM in patients with cN0 PTC, we also created a 24-point risk score model based on these 10 independent risk factors from multivariate analysis to predict whether such patients have CLNM. The predictive model can not only observe the influence of each independent risk factor on the incidence of CLNM, but also quickly assess the incidence of CLNM in individual cN0 PTC patients. The ROC curve of this risk scoring model showed that the AUC for predicting CLNM is 0.904 (95\% CI: 0.880 to 0.928 , $\mathrm{P}<0.001)$, indicating that the model has a high predictive effect. The incidence of CLNM in patients with cNO PTC scores $\geq 14$ was significantly higher than that of patients with cN0 PTC scores $\leq 13$ (87.96\% vs. $23.04 \%, \mathrm{P}<0.001)$. The ROC curve of the test model indicated that the AUC for predicting CLNM was 0.956 (95\% CI: 0.915 to 0.996, $\mathrm{P}<0.001$ ), which further proves the rationality of this model to predict CLNM. Such a risk scoring model is simple and effective, and can better guide clinical work. Clinicians use a relatively objective way to identify high-risk populations of CLNM and provide personalized treatment for these patients.

In summary, our study found that male gender, age 
$<45$ years old, tumor maximum diameter $\geq 1.0 \mathrm{~cm}$, tumor location: middle/lower poles of the thyroid gland, multifocality, extrathyroidal extension, and some ultrasound features, such as intra-nodular vascularity, microcalcification, irregular shape, infiltrative margin, are independent risk factors for CLNM in patients with cN0 PTC. Considering that CLNM is an independent risk factor for recurrence. We have established a 24-point risk scoring model to better guide the surgical methods of patients with cN0 PTC. For cN0 PTC patients with scores $\geq 14$, even if no CLNM is found before surgery, routine preventive central lymph node dissection is recommended. In addition, for $\mathrm{cN} 0 \mathrm{PTC}$ patients with a score of fewer than 14 points, it is recommended to perform FNA before surgery, carefully assess the condition of the central lymph nodes, and then select the best surgical plan based on the results of the assessment.

\section{Acknowledgments}

Funding: This work was supported by grants from the Research Project of Maternal and Child Health of Jiangsu Province (F201953), and the Science and Technology Project of Nantong (JC2020067) to Z He.

\section{Footnote}

Reporting Checklist: The authors have completed the STARD reporting checklist. Available at https://gs.amegroups.com/ article/view/10.21037/gs-21-906/rc

Data Sharing Statement: Available at https://gs.amegroups. com/article/view/10.21037/gs-21-906/dss

Conflicts of Interest: All authors have completed the ICMJE uniform disclosure form (available at https://gs.amegroups. com/article/view/10.21037/gs-21-906/coif). The authors have no conflicts of interest to declare.

Ethical Statement: The authors are accountable for all aspects of the work in ensuring that questions related to the accuracy or integrity of any part of the work are appropriately investigated and resolved. The study was conducted in accordance with the Declaration of Helsinki (as revised in 2013). This study was approved by the Ethics Committee of Affiliated Hospital of Nantong University (ID: 2013-67). Informed consent was provided by all participants.
Open Access Statement: This is an Open Access article distributed in accordance with the Creative Commons Attribution-NonCommercial-NoDerivs 4.0 International License (CC BY-NC-ND 4.0), which permits the noncommercial replication and distribution of the article with the strict proviso that no changes or edits are made and the original work is properly cited (including links to both the formal publication through the relevant DOI and the license). See: https://creativecommons.org/licenses/by-nc-nd/4.0/.

\section{References}

1. Seib CD, Sosa JA. Evolving Understanding of the Epidemiology of Thyroid Cancer. Endocrinol Metab Clin North Am 2019;48:23-35.

2. Schneider DF, Chen H. New developments in the diagnosis and treatment of thyroid cancer. CA Cancer J Clin 2013;63:374-94.

3. Guo Z, Ge M, Chu YH, et al. Recent Advances in the Classification of Low-grade Papillary-like Thyroid Neoplasms and Aggressive Papillary Thyroid Carcinomas: Evolution of Diagnostic Criteria. Adv Anat Pathol 2018;25:263-72.

4. Rubinstein JC, Dinauer C, Herrick-Reynolds K, et al. Lymph node ratio predicts recurrence in pediatric papillary thyroid cancer. J Pediatr Surg 2019;54:129-32.

5. Zhao H, Huang T, Li H. Risk factors for skip metastasis and lateral lymph node metastasis of papillary thyroid cancer. Surgery 2019;166:55-60.

6. Eltelety AM, Terris DJ. Neck Dissection in the Surgical Treatment of Thyroid Cancer. Endocrinol Metab Clin North Am 2019;48:143-51.

7. Medas F, Canu GL, Cappellacci F, et al. Prophylactic Central Lymph Node Dissection Improves DiseaseFree Survival in Patients with Intermediate and High Risk Differentiated Thyroid Carcinoma: A Retrospective Analysis on 399 Patients. Cancers (Basel) 2020;12:1658.

8. Nixon IJ, Wang LY, Ganly I, et al. Outcomes for patients with papillary thyroid cancer who do not undergo prophylactic central neck dissection. Br J Surg 2016;103:218-25.

9. Huang $\mathrm{H}, \mathrm{Wu} \mathrm{L}$, Liu $\mathrm{W}$, et al. Long-term outcomes of patients with papillary thyroid cancer who did not undergo prophylactic central neck dissection. J Cancer Res Ther 2020;16:1077-81.

10. Wu X, Li BL, Zheng CJ, et al. Predictive factors for central lymph node metastases in papillary thyroid microcarcinoma. World J Clin Cases 2020;8:1350-60. 
11. Ataş H, Akkurt G, Saylam B, et al. Central neck dissection is an independent risk factor for incidental parathyroidectomy. Acta Chir Belg 2021;121:36-41.

12. Park I, Her N, Choe JH, et al. Management of chyle leakage after thyroidectomy, cervical lymph node dissection, in patients with thyroid cancer. Head Neck 2018;40:7-15.

13. National Cancer Institute. Surveillance Epidemiology and End Results (SEER) Program. Bethesda, MD,USA: National Cancer Institute, 2006.

14. Hu J, Yuan IJ, Mirshahidi S, et al. Thyroid Carcinoma: Phenotypic Features, Underlying Biology and Potential Relevance for Targeting Therapy. Int J Mol Sci 2021;22:1950.

15. Stack BC Jr, Ferris RL, Goldenberg D, et al. American Thyroid Association consensus review and statement regarding the anatomy, terminology, and rationale for lateral neck dissection in differentiated thyroid cancer. Thyroid 2012;22:501-8.

16. Viola D, Materazzi G, Valerio L, et al. Prophylactic central compartment lymph node dissection in papillary thyroid carcinoma: clinical implications derived from the first prospective randomized controlled single institution study. J Clin Endocrinol Metab 2015;100:1316-24.

17. Glover AR, Gundara JS, Norlén O, et al. The pros and cons of prophylactic central neck dissection in papillary thyroid carcinoma. Gland Surg 2013;2:196-205.

18. Ma B, Wang Y, Yang S, et al. Predictive factors for central lymph node metastasis in patients with $\mathrm{cN} 0$ papillary thyroid carcinoma: A systematic review and meta-analysis. Int J Surg 2016;28:153-61.

19. Jiang LH, Yin KX, Wen QL, et al. Predictive Riskscoring Model For Central Lymph Node Metastasis and Predictors of Recurrence in Papillary Thyroid Carcinoma. Sci Rep 2020;10:710.

20. Liu C, Xiao C, Chen J, et al. Risk factor analysis for predicting cervical lymph node metastasis in papillary thyroid carcinoma: a study of 966 patients. BMC Cancer 2019;19:622.

21. Zhan J, Diao X, Chen Y, et al. Predicting cervical lymph

Cite this article as: Zhong X, Lu Y, Yin X, Wang Q, Wang F, He Z. Prophylactic central lymph node dissection performed selectively with cN0 papillary thyroid carcinoma according to a risk-scoring model. Gland Surg 2022;11(2):378-388. doi: 10.21037 /gs-21-906 node metastasis in patients with papillary thyroid cancer (PTC) - Why contrast-enhanced ultrasound (CEUS) was performed before thyroidectomy. Clin Hemorheol Microcirc 2019;72:61-73.

22. Tang T, Li J, Zheng L, et al. Risk factors of central lymph node metastasis in papillary thyroid carcinoma: A retrospective cohort study. Int J Surg 2018;54:129-32.

23. Luo X, Wang J, Xu M, et al. Risk model and risk stratification to preoperatively predict central lymph node metastasis in papillary thyroid carcinoma. Gland Surg 2020;9:300-10.

24. Kim K, Shim SR, Lee SW, et al. Diagnostic values of F-18 FDG PET or PET/CT, CT, and US for Preoperative Lymph Node Staging in Thyroid Cancer: A Network Meta-Analysis. Br J Radiol 2021;94:20201076.

25. Jang JY, Kim DS, Park HY, et al. Preoperative serum VEGF-C but not VEGF-A level is correlated with lateral neck metastasis in papillary thyroid carcinoma. Head Neck 2019;41:2602-9.

26. Shin HS, Na DG, Paik W, et al. Malignancy Risk Stratification of Thyroid Nodules with Macrocalcification and Rim Calcification Based on Ultrasound Patterns. Korean J Radiol 2021;22:663-71.

27. Li F, Pan D, He Y, et al. Using ultrasound features and radiomics analysis to predict lymph node metastasis in patients with thyroid cancer. BMC Surg 2020;20:315.

28. Liu J, Zheng D, Li Q, et al. A predictive model of thyroid malignancy using clinical, biochemical and sonographic parameters for patients in a multi-center setting. BMC Endocr Disord 2018;18:17.

29. Watanabe K, Igarashi T, Ashida H, et al. Diagnostic value of ultrasonography and TI-201/Tc-99m dual scintigraphy in differentiating between benign and malignant thyroid nodules. Endocrine 2019;63:301-9.

30. Wang XQ, Wei W, Wei X, et al. Study on the relationship between ultrasonographic features of papillary thyroid carcinoma and central cervical lymph node metastasis. Zhonghua Zhong Liu Za Zhi 2018;40:196-200.

(English Language Editor: J. Jones) 